
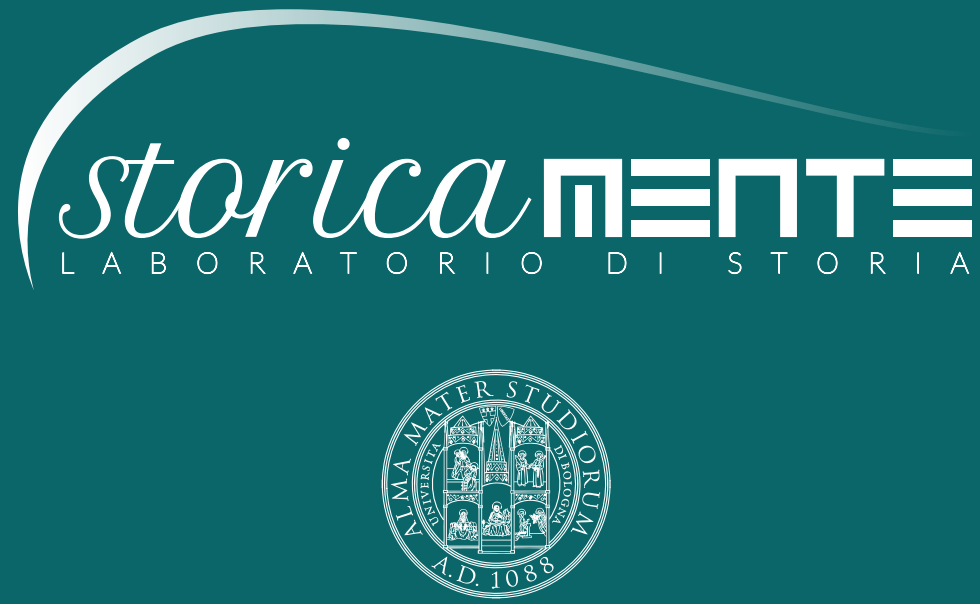

ALMA MATER STUDIORUM

Università di Bologna

Dipartimento di Storia Culture Civiltà

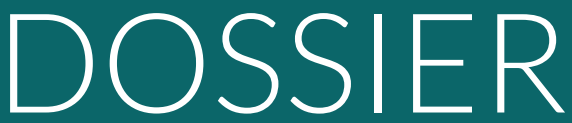

Representations of Fear in History

Empirical / Practical evidence and Methodological Issues 


\section{STORICAMENTE.ORG}

\section{Laboratorio di Storia}

Tommaso Gnoli

Metus Gallicus: "metus” come spinta al cambiamento

Numero 11 - 2015

ISSN: $1825-411 \mathrm{X}$

Art. 20

pp. 1-16

DOI: $10.12977 /$ stor605

Editore: BraDypUS

Data di pubblicazione: 31/12/2015

Licenza: CC BY-NC-ND 4.0 International

Articolo sottoposto a revisione paritaria a doppio cieco

Dossier: Representations of Fear in History: Empirical / Practical evidence and Methodological Issues edited by Vito Gironda, Marica Tolomelli 


\title{
Metus Gallicus: "metus" come spinta al cambiamento
}

\author{
TOMMASO GNOLI \\ Univ. Bologna, \\ Dipartimento di Storia Culture Civiltà,
}

A partire dal sacco di Roma da parte di Brenno, la coscienza nazionale romana si è costituita attorno al metus Gallicus, che ha avuto un ruolo importante anche per determinare l'identità romana e plasmare il concetto stesso di Italia. S'indaga come, partendo da questa paura, anche rituali religiosie perfino istituzioni qualil'esercito abbiano conosciuto mutazioni e cambiamenti. Since the sack of Rome by the Gauls of Brennus the national Roman self-consciousness had been built upon the concept of metus Gallicus, which played a central role also in shaping Roman identity and the idea of Italy. This article aims at explaining how religious rituals and civic institutions such as the army underwent changes because of this very fear.

L'episodio del sacco di Roma da parte dei Galli del 386 a.C. costituisce uno dei momenti più importanti di tutta la fase arcaica della storia di Roma. Nonostante la notorietà dell'evento e le efficaci e informate analisi che ne sono state date ancora in tempi molto recenti ${ }^{2}$, mi sembra

1 Le fonti principali sull'episodio sono Liv. 5,33-50, Dion. Hal. A.R. 13,6 sgg.; Plut. Cam.15-30; Diod. 14,113-117. A queste si affiancano altre fonti meno estese e dettagliate: Pol. 1,6,2-4; 2,18,1-4; 2.22,4-5; App. Gall., fr. 1,1; 2-9; Ital. fr. 8,2; Dio fr. 25, Zon. 7,23, oltre ai brevi resoconti di Flor. 1,7,13-19; Eutr. 1,20; De Vir. Ill. 23. Ognuna di queste fonti presenta sue proprie caratteristiche e varianti, efficacemente analizzate nei classici e ancora oggi fondamentali Mommsen 1879a; 1879b.

2 Per limitarsi agli ultimi lavori a me noti: Williams 2001, Briquel 2008, Delfino 2009, Richardson 2012, Roberto 2012. 
sia il caso di richiamare in breve i tratti fondamentali di questa vicenda, visto il carattere ampiamente diacronico di questo Dossier. Nel corso del quinto secolo avanti Cristo una grandiosa Volkerwanderung ebbe luogo nell'Italia settentrionale [Polyb. 2,17]. Gli Etruschi vennero cacciati da gran parte della Pianura Padana orientale. Felsina divenne Bononia, dal nome di una delle tribù d'invasori, i Boi. L'ondata gallica si diffuse nei primi decenni del quarto secolo lungo la costa adriatica fino a Sena Gallica (Senigallia), un'altra località che prese il nome da una tribù d'invasori, questa volta i Senones ${ }^{\text {: }}$ il paesaggio etnico dell'Italia centro-settentrionale mutò profondamente.

A partire dalle coste adriatiche e dalla valle del Po recentemente occupata, i nuovi padroni s'interessarono ben presto alla fertile valle del Tevere e al mosso e ricco paesaggio di Umbria ed Etruria. La tradizione romana, sempre alla ricerca d'identificare un chiaro e riconoscibile casus belli, inventò una strana storia: un fuoriuscito di Clusium, un certo Arunte, avrebbe attirato il rapace interesse della rude gente gallica, mostrando loro due ben rinomati prodotti Italici: olio d'oliva e vinot. L'orda gallica invase l'Umbria a Clusium, proprio nel momento in cui un'ambasceria proveniente da Roma veniva ricevuta nella città etrusca. In conformità con i caratteri arcaici dello Stato romano, questa ambasceria sembra una sorta di questione di famiglia - o meglio di gens: la tradizione afferma che gli ambasciatori erano tutti e tre Fabii. Durante

\section{Zecchini 2006.}

4 La storia è riferita con diversi livelli di dettaglio da Liv. 5,33,3-4; Dion. Hal., A.R. 13,10-11; Plut., Cam. 15,3-4. Una tradizione con protagonisti diversi è conservata da Plinio, N.H.12,5, secondo il quale responsabile dell'invasione dei Galli sarebbe stato un certo Helicus, un Gallo che viveva tra gli Elvezi, che si sarebbe trasferito per qualche tempo a Roma per praticare la sua professione di fabbro. Ne avrebbe riportato ficum siccam et uvam oleique ac vini promissa che avrebbero oltremodo ingolosito i suoi compagni. È difficile dire quando sia nata questa variante, con la responsabilità scaricata sugli Elvezi: è possibile pensare agli anni immediatamente successivi alla guerra Cimbrica - all'inizio del primo secolo a.C. -, oppure a ridosso dell'intervento di Cesare in Gallia. Ha comunque ragione Williams 2001 [108] a voler vedere nella variante pliniana un caso di attualizzazione della minaccia gallica nella Roma del primo secolo. 
i combattimenti a Clusium un ambasciatore romano avrebbe ucciso un nobile gallico, contravvenendo le leggi sacre che imponevano agli ambasciatori di non prender parte ai combattimenti. I Galli chiesero soddisfazione a Roma ma, nonostante il senato avesse stigmatizzato il comportamento dei Fabii, il popolo si rifiutò di consegnare i tre responsabili ai Galli, ed anzi li elesse per tutta risposta alla carica di tribuni militari con potestà consolare.

L'irresponsabile comportamento dei Fabii e del popolo attrasse dunque su Roma l'ira di Brenno, il re dei Galli. Il 18 luglio 386 un esercito romano posto sotto il comando dei tre tribuni militari ${ }^{6}$ venne annientato sul fiume Allia, da tempo identificato con il Fosso Maestro, al km 18,3 della Salaria, a nord di Roma. Nei giorni successivi i Galli invasero l'Urbs, costringendo i difensori a barricarsi sul Campidoglio mentre il resto della città veniva abbandonato al saccheggio da parte degli in-

5 La data della battaglia coincide perfettamente con un'altra cocente sconfitta subita da Roma: quella avvenuta circa cento anni prima ad opera dei Veienti sul fiume Cremera [Liv. 6,1,12]. La coincidenza tra il dies Alliensis e il dies Cremerensis è un'invenzione riconducibile al filone storiografico facente capo alla gens Fabia, come ha brillantemente mostrato Santo Mazzarino 1966, II.1, 248-249. In una recente monografia Richardson [2012] ha utilizzato le vicende della gens Fabia e della sua opposizione ai Galli come un caso di studio per l'argomento più generale del ruolo che la mentalità romana di età repubblicana attribuiva alle varie gentes. I comportamenti degli individui erano resi nella tradizione storiografica secondo delle caratteristiche ritenute comuni o comunque prevalenti tra i membri di quel determinato clan gentilizio secondo dei criteri di probabilità o verosimiglianza che spesso poco avevano $\mathrm{a}$ che vedere con l'effettivo svolgimento dei fatti (stock characters).

6 La questione del comando dell'esercito nella battaglia del fiume Allia ha anch'essa provocato consistenti varianti nella tradizione. Livio ne conosce due: la vulgata attribuiva senz'altro il comando ai tre neo-eletti tribuni militari già ambasciatori a Clusium, cioè i tre fratelli figli di M. Fabio Ambusto [5,37-38]. Accanto a questa tradizione, però, ve n'era anche un'altra che diceva che il comando dell'esercito in quell'occasione era detenuto da un quarto tribuno militare, Q. Sulpicio $[6,1,12]$. Quest'ultima versione, riferita come secondaria da Livio, è riportata in forma più estesa da Macrobio [Sat. 1,16,21-24] e da Gellio [N.A. 5,17,1-2] dove si dice che risalirebbe a Cassio Hemina e a Cn. Gellio. Sul valore eziologico di questa variante, che mal si coniuga con quanto si conosce sulla tradizione storiografica di stampo annalistico, cfr. da ultimo Richardson 2012, 119-120. 
vasori, che sarebbero rimasti in possesso dell'Urbe per un periodo di ben sette mesi [Polyb. 2,22,5]. Durante i ripetuti tentativi di conquista della rocca capitolina la tradizione colloca vari episodi d'eroismo, che acquisirono subito valore topico, in particolare l'impresa dello strenuo difensore Marco Manlio, che prese quindi il nome di Capitolino. A questo punto però la storia si complica, perché sono pervenute diverse versioni di quanto seguì immediatamente. La tradizione vulgata, quella maggiormente patriottica e più favorevole a Roma, afferma che i Galli vennero cacciati con la forza dal dittatore Marco Furio Camillo, che recuperò la città senza il pagamento di alcun riscatto: riunitisi assediati e assedianti per concordare l'entità del tributo, infatti, i Galli si sarebbero presentati con le bilance per pesare l'oro pattuito (mille libbre) palesemente truccate. All'insolente affermazione di Brenno che voleva sanzionare il diritto del più forte ("vae victis») si oppose con efficacia il dittatore Marco Furio Camillo, che si materializza all'improvviso nel racconto liviano, e costrinse dapprima i Galli a ritirarsi a mani vuote, quindi li sterminò in una splendida battaglia .

Quasi tutte le altre versioni sapevano invece del tributo effettivamente pagato dalla città per la sua liberazione's . Le famose mille libbre d'oro sarebbero poi state successivamente variamente recuperate grazie all'intervento sempre di Marco Furio Camillo, che avrebbe sconfitto successivamente i Galli ebbri di vittoria ${ }^{\circ}$. Oppure, con un'altra variante, sarebbe stato recuperato solamente trecento anni dopo, all'inizio del primo secolo avanti Cristo, ad opera di Marco Livio Druso [Suet., Tib. 3] ${ }^{10}$.

Tutte queste, comunque, sembrano essere tradizioni successive, mentre

7 Di questi si è occupato Ungern-Stemberg 2000.

8 L'eccezione più notevole è costituita da Polibio, che non conosce né sconfitta dei Galli, né recupero del riscatto.

9 Liv. 5,33-50, la migliore rappresentazione della vulgata storiografica.

10 Su questa tradizione cfr. Williams 2001, 42. 
più antica, e certamente degna di maggior considerazione, appare una versione sopravvissuta in modo sporadico in fonti greche a noi trasmesse da Plutarco (soprattutto nella Vita di Camillo) e in Diodoro Siculo" ${ }^{11}$. Plutarco afferma che secondo Aristotele: «Roma era stata conquistata dai Celti e Lucio l'aveva salvata» [Plut., Cam. 22,4 = F 610 Rose]. Marta Sordi [1960, 62-72] ha molto correttamente restituito il senso di questa notizia: il Lucio di cui parla Aristotele (Plutarco) non è un alter ego di Marco Furio Camillo, il salvatore di Roma della tradizione romana successiva, ma è un altro personaggio, certamente il plebeo Lucius Albinius al quale la tradizione affida il ruolo di salvatore dei sacra Urbis, cioè di quegli arredi sacri essenziali alla religiosità romana, tra i quali il Palladio, che vennero salvati assieme al sacro collegio delle Vergini Vestali trasportandolo, tutto o in parte ${ }^{12}$, nella vicina Caere. Il Lucius (Albinius) di Aristotele (Plutarco) avrebbe quindi salvato Roma nel senso che, secondo una successiva tradizione, ne avrebbe salvato i sacra, mentre l'effettivo salvataggio dell'Urbe sarebbe stato opera dei vari Tito Manlio e, soprattutto, Furio Camillo, come s'è visto. Mi sembra del tutto corretta l'analisi di Mazzarino [1966, 250-252], che, sulla scorta di Sordi, individua nella tradizione di Aristotele la versione originaria, contenuta negli Annali dei pontefici, prima delle contaminazioni liviane. Questa versione non conosce riscatti né miracolosi recuperi di quanto pagato, conosce solamente un "salvataggio metafisico" dell’Urbe, che venne

11 Williams 2001, 146: «As Rome itself grew in importance in the fourth and third centuries, so interest in its history grew among the Greeks, as the fragmentary remains of Timaeus' detailed investigations into Roman history and religion suggest».

12 Una parte della tradizione afferma che le Vergini Vestali, prima d'incamminarsi verso Caere, avevano già provveduto a seppellire in luogo sicuro parte dei sacra. Il "salvataggio" di Lucius Albinius, quindi, si sarebbe via via depotenziato, limitandosi ad offrire il suo carro per trasportare le vergini e parte dei sacra, dopo averne fatto discendere la sua famiglia. Il depotenziamento del ruolo di questo personaggio nella vicenda del sacco gallico, fino a renderlo un mero exemplum virtutis, e specificamente pietatis, sembra poter esser messo in relazione con la di poco successiva "serrata del patriziato", che tese a limitare il ruolo dei plebei nelle magistrature dopo l'approvazione delle leges Liciniae Sextiae. 
invece concretamente conquistata e saccheggiata dai barbari vincitori. Se questi furono gli accadimenti come sono stati riferiti da una tradizione storica molto più recente, resta il fatto che «il capitolo essenziale per ogni studio sulla formazione dell'annalistica romana è [...] la formazione della tradizione sull'incendio gallico» [Mazzarino 1966, II 284]. In effetti, questo episodio si pone al crocevia di una molteplicità di problemi che possono così essere riassunti:

- la creazione delle diverse tradizioni storiche (annalistiche)

- il conflitto tra patrizi e plebei

- le relazioni tra Romani e Galli (anche nella più ampia, e impropria, accezione di 'popoli settentrionali')

- la conquista (e l'idea) dell'Italia

- l'evoluzione delle magistrature romane

- l'evoluzione della religione romana ${ }^{13}$.

Molti di questi temi, come s'è detto, vennero strutturati dalla tradizione attorno alla figura, ricca di tratti mitologici ${ }^{14}$, di Marcus Furius Camillus, la cui vicenda è stata definita «Die verlogenste aller römischen Legenden, die Camillusfabel» [Mommsen 1899, 1018 n. 2], o ancora «the lifeless figure of Camillus, the most artificially contrived of all Rome's heroes» [Cornell 1995, 317].

Il terrore dei Galli, il metus Gallicus, dopo questi eventi rimase impresso nella coscienza collettiva dei Romani come un marchio evidente. Quando, nel 225 a.C. la tribù germanica dei Gaesati passò le Alpi, a

\section{Cfr. Williams 2001, 141.}

14 La tradizione relativa a Marco Furio Camillo, resa estremamente complessa anche dalla qualità delle fonti che lo riguardano - in primo luogo Plutarco - non viene presa in considerazione in questo contributo. Del resto la figura di questo eroe dai tratti decisamente divini - un nuovo Romolo - ha un'importanza decisiva per il tema della rinascita dell'Urbe dopo il saccheggio, così come - da un punto di vista ideologico - su quello della titolatura che verrà concessa dal senato di Roma a Giulio Cesare Ottaviano nel 27 a.C. Di questi si è ripetutamente occupato Georges Dumézil, di cui basterà ricordare, in questa sede, Dumézil 1973; 1974, 403-405; 1980 passim. Nella sterminata bibliografia molto utili Bruun 2000 e von Ungern-Stemberg 2000. 
Roma si diffuse una vera e propria psicosi. Bellen [1985] ha notato che di lì a pochi anni, durante la Seconda Guerra Punica (219-202 a.C.) il metus Gallicus si sarebbe saldato con il metus Punicus, generando ciò che Polibio chiamò '̌́ $\xi \omega \theta \varepsilon v$ póßos, Sallustio e Cicerone metus hostilis. Da un punto di vista meramente politico questo metus divenne quindi il più potente motore per l'imperialismo romano: la categoria di metus/ póßos mutò in continuazione la sua determinazione, volta a volta assumendo quella degli avversari del momento: si veda la bella analisi del

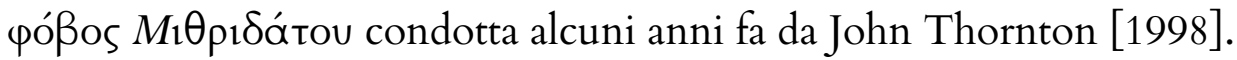
Sarebbe tuttavia sbagliato voler derubricare il metus Gallicus ad un mero espediente per la conquista dell'Italia settentrionale, come mostrerà ciò che segue.

Quando, alla fine del quarto secolo dopo Cristo o, a mio avviso più plausibilmente, all'inizio del quinto ${ }^{15}$, un anonimo autore sotto lo pseudonimo di Flavius Vopiscus scrisse la biografia di un personaggio ai suoi occhi eccezionale nella Storia romana, l'imperatore Probo, egli affermò che, senza la sua opera biografica, si rischiava di perdere «il ricordo di un uomo tanto grande che non ebbe eguali né nelle guerre puniche, né nelle invasioni galliche, né nelle sommosse pontiche, né negli intrighi ispanici» (tanti viri et talis historia, qualem non habent bella Punica, non terror Gallicus, non motus Pontici, non Hispaniensis astutia - HA, Probus 1,4). Nella costruzione altamente retorica costituita da binomi formati da un sostantivo e da un aggettivo derivato da un etnonimo, la storia antica di Roma veniva quindi riassunta, nel bel mezzo della tarda antichità, nei quattro grandi eventi delle guerre puniche, delle guerre

15 Non è assolutamente questa la sede di affrontare nemmeno di sfuggita la spinosissima questione della data di composizione della cosiddetta Historia Augusta, una raccolta apocrifa di biografie imperiali variamente datata tra fine quarto e primi decenni del quinto - altre possibili datazioni, in particolare quella tra terzo e quarto secolo, sono oramai quasi scomparse dal panorama scientifico attuale. La mia posizione al riguardo ancora una volta coincide con quella più volte espressa, tra gli altri, da Santo Mazzarino [1966; 1973; 1974], uno tra i principali fautori di una datazione bassa dell'opera, posizione attualmente minoritaria tra gli studiosi di antichistica. 
contro i Galli, di quelle contro Mitridate e della difficile conquista della Spagna. Vi si possono vedere i quattro punti cardinali, oppure ancora la conquista dell'Italia e dei tre continenti che costituivano l'ecumene, ma è veramente interessante l'attenta variatio dei sostantivi. La categoria del bellum riguarda solo Cartagine. Il ricordo dei cosiddetti "vespri Efesii" e il connesso sterminio degli Italici nelle città d'Asia relegava irrimediabilmente le guerre Mitridatiche nella categoria del motus. Più enigmatica è la categoria dell'astutia usata per caratterizzare le guerre in Spagna, molto probabilmente scelta in relazione alla vicenda di Sertorio e della stretta connessione esistente tra queste lunghe e sanguinose guerre e le contemporanee lotte civili a Roma. Per quanto riguarda i Galli, però, non ci sono dubbi: la categoria del terror è solamente un'amplificazione del metus di cui si parla. A scanso di equivoci, la collocazione del metus in seconda posizione, dopo le guerre puniche, chiarisce ulteriormente, se ve ne fosse bisogno, che deve intendersi come generico terrore dei popoli settentrionali, e non limitato ai Galli di Brenno.

Non molti anni dopo il traumatico sacco di Roma da parte di Alarico (410) due esponenti delle gerarchie ecclesiastiche, ognuno seguendo le proprie, diverse, capacità intellettuali, offrirono la stessa spiegazione e la medesima descrizione degli eventi: ciò che era da poco accaduto e che aveva profondamente colpito i contemporanei, non era nulla in paragone a quanto era accaduto in un passato lontano, e ormai quasi mitico.

Oros., Hist. adv. pag. 2,19,12-15: En tempora quorum conparatione praesentia ponderantur; en, quibus recordatio suspirat; en, quae incutiunt de electa uel potius de neglecta religione paenitentiam! Reuera pares sunt et conferuntur inter se hae duae captiuitates! illa sex mensibus desaeuiens et tribus diebus ista transcurrens; Galli exstincto populo Vrbe deleta ipsum quoque Romae nomen in extremis cineribus persequentes, et Gothi relicta intentione praedandi ad confugia salutis, hoc est sanctorum locorum, agmina ignara cogentes; ibi uix quemquam inuentum senatorem qui uel absens euaserit, hic uix quemquam requiri qui forte ut latens perierit. Recte sane conpararim hunc fuisse ibi seruatorum numerum qui hic fuerit perditorum. Plane quod re proditur et fatendum est: in hac clade praesenti plus Deum saeuisse, homines 
minus, cum, peragendo ipse quod illi non inpleuissent, cur eos miserit, demonstrauit. Quippe cum supra humanas uires esset incendere aeneas trabes et subruere magnarum moles structurarum, ictu fulminum forum cum imaginibus uanis quae superstitione miserabili uel Deum uel hominem mentiuntur, abiectum est; horumque omnium abominamentorum, quod inmissa per hostem flamma non adiit, missus e caelo ignis euertit ${ }^{16}$.

Gli stessi temi, ma con accenti molto diversi, si possono riscontrare in Agostino:

Aug., De civ. Dei, 3,29: quae rabies exterarum gentium, quae saeuitia barbarorum huic de ciuibus uictoriae ciuium comparari potest? quid Roma funestius taetrius amariusque uidit, utrum olim Gallorum et paulo ante Gothorum inruptionem an Marii et Sullae aliorumque in eorum partibus uirorum clarissimorum tamquam suorum luminum in sua membra ferocitatem? Galli quidem trucidauerunt senatum, quidquid eius in Urbe tota praeter arcem Capitolinam, quae sola utcumque defensa est, reperire potuerunt; sed in illo colle constitutis auro uitam saltem uendiderunt, quam etsi ferro rapere non possent, possent tamen obsidione consumere: Gothi uero tam multis senatoribus pepercerunt,

16 «Ecco $\mathrm{i}$ tempi, in confronto ai quali si valutano gli avvenimenti presenti! ecco le cose al cui ricordo si sospira! ecco i fatti che dovrebbero farci pentire di aver scelto una religione, o meglio, di averne tralasciata un'altra! È proprio il caso di considerare uguali questi due momenti in cui Roma fu occupata dai nemici e di metterli sullo stesso piano! La prima occupazione infierì per sei mesi, la seconda trascorse in tre giorni; i Galli annientarono il popolo romano, distrussero la città e perseguitarono lo stesso nome di Roma, non contentandosi di averla ridotta in cenere; i Goti, invece, deposta la cupidigia di predare, spinsero le schiere ignare dei cittadini a cercare rifugio e salvezza nei luoghi sacri; allora si trovò a stento qualche senatore che riuscì a scampare, non trovandosi in città; ora a malapena si è potuto trovare qualcuno che sia morto nascondendosi. Potrei giustamente dire che il numero di coloro che si salvarono in quella occasione fu uguale al numero dei morti in questa. Si deve riconoscere, come del resto dimostrano i fatti, che nella sciagura odierna ha infierito più Dio degli uomini, giacché, portando a termine egli stesso ciò che i barbari non avevano compiuto, ha reso evidenti le ragioni per cui li ha mandati. Infatti essendo superiore alle forze dell'uomo distruggere con l'incendio le travi di bronzo e abbattere le grandi moli degli edifici, Dio ha colpito con i fulmini il Foro e le sue vane statue, le quali con miserabile superstizione ci danno un'immagine menzognera sia della divinità che dell'uomo: e così tutte quelle cose abominevoli, che le fiamme scagliate dai nemici non poterono toccare, sono state distrutte dal fuoco celeste» (trad. A Bartalucci). 
ut magis mirum sit quod aliquos peremerunt ${ }^{17}$.

Ci sarebbe molto da discutere in questo brano agostiniano, a partire dalla rapidissima ripresa dell'apologo di Menenio Agrippa sulle varie parti che, insieme, costituiscono l'organismo statale ${ }^{18}$. Qui i due episodi dei saccheggi dell'Urbe sono posti in una graduatoria di orrori che vede incontrastato, al vertice, l'abominio delle guerre civili.

Il sacco di Roma da parte di Brenno divenne la pietra di paragone sulla quale valutare il sacco di Alarico. Ottocento anni dopo, gli eventi sopra riportati erano ancora ben presenti nella memoria storica dei contemporanei, o, meglio, questi eventi, così com'erano riportati dalla tradizione storica, finirono con il costituire la memoria storica dei Romani della tarda antichità. L'identità si determina sulla base dell'alterità, e il sacco dei Galli fu uno snodo fondamentale in questo processo identitario. La imagined community di Roma, la sua identità culturale, si costruì in larga misura attorno a questo evento traumatico d'inizio quarto secolo avanti Cristo.

L'evento lasciò tracce profonde anche nei culti ${ }^{19}$. Per ben tre volte, nel 228, nel 216 e nel 113, le fonti letterarie riferiscono che, dopo aver

17 «Quale furore di genti straniere, quale crudeltà di barbarie si può paragonare a questa vittoria di cittadini su cittadini [il riferimento è alla marcia su Roma di Silla]? Che cosa ha visto Roma di più efferato, macabro e desolante, forse l'antico saccheggio dei Galli e il recente dei Goti o piuttosto la violenza di Mario e Silla e degli altri uomini eminenti nei rispettivi partiti? Fu come la violenza degli occhi di Roma contro le sue membra. I Galli uccisero i senatori ovunque li avessero trovati in tutta Roma, fuorché nella rocca del Campidoglio che comunque era la sola ad essere difesa. A coloro però che si trovavano su questo colle permisero per lo meno di riscattare la vita con l'oro, e sebbene non potessero toglierla con le armi, avrebbero potuto farla deperire con un lungo assedio. I Goti poi hanno risparmiato tanti senatori che farebbe meraviglia se ne hanno uccisi alcuni. Invece Silla, e Mario era ancora vivo, s'insediò come vincitore sul Campidoglio, che fu rispettato dai Galli, per decretare le carneficine [...] L'ultima tavola di Silla, inoltre, per non parlare di molte altre uccisioni, mandò a morte più senatori di quanti i Goti riuscirono a derubare» (trad. D. Gentili).

18 Cfr. Liv. 2, 32.

19 Cfr. in particolare Ungern-Sternberg 2000. 
consultato i libri Sibillini, i Romani procedettero a un rito estremamente crudele: seppellirono vivi nel Foro Boario una coppia di Galli e una coppia di $\mathrm{Greci}^{20}$. Livio tentò di giustificare il comportamento dei suoi antenati dichiarando a chiare lettere che quel rito non era affatto Romano: minime Romano sacro, ma, ovviamente, mentiva: il rito era perfettamente Romano, come testimonia senza possibili ambiguità lo strano assortimento delle coppie. Se infatti è chiaro il motivo che aveva condotto al seppellimento della coppia di Galli, cosa indusse i Romani a fare altrettanto con una coppia di Greci? Roma non fu mai in guerra contro queste due etnie contemporaneamente. Oltretutto l'andamento stesso delle guerre combattute in Italia contro le città greche è insufficiente a giustificare, in qualche modo, una ritualità così drammatica e ripugnante. D'altronde, proprio la crudezza di tali riti rende quantomeno improbabile pensare a un'invenzione tardiva. Sia Augusto Fraschetti [1981] sia Andrea Giardina [1997] hanno certamente ragione ad affermare che la presenza nel rito di Galli e Greci rappresenta la miglior garanzia di autenticità del rito stesso. In queste pratiche rituali nel Foro Boario si ha un antico ricordo di un periodo in cui il concetto d'Italia coincideva con lo spartiacque appenninico: Galli e Greci erano i più importanti ethne insediati nei territori transappenninici ${ }^{21}$. Tra i molti motivi d'interesse offerti da questo terribile rito vi è quindi proprio il ricordo di un'Italia 'cis-appenninica', come scrisse Giardina, che in questo senso sembra riconnettersi con una delle tante idee-guida del suo maestro, Santo Mazzarino. Scrivendo, nel 1944, il suo Dalla monarchia allo stato repubblicano, egli avanzò la teoria dell'esistenza, si-

20 Le fonti al riguardo sono: Plut., Marc. 3 e Oros., 4,13,1-4 per il 228; Liv. 22,57,16 (minime Romano sacro) per il 216, l'annus horribilis della Seconda Guerra Punica; Plut., Quaest. Romanae 284; Pl. N.H. 28, XX; Plut. Marius 27,6-9 per il 113. Su tutte queste fonti cfr. innanzi tutto Fraschetti 1981, che qui si segue.

21 Sull'idea d'Italia delimitata dalla dorsale appenninica le fonti principali sono Liv. 5,33,9 e Diod.14,113,2. Importanti considerazioni sul tema del polimorfismo del concetto d'Italia nel recentissimo Russo 2012. 
gnificativamente operativa fino a tutto il quarto secolo avanti Cristo, di quella che chiamò una 'koinè (centro) italica' ${ }^{22}$ in nessun caso mai limitata ai soli elementi romano-etruschi, bensì inclusivi, volta a volta e non senza selezioni, molti elementi del complesso mosaico culturale italico. Ha ragione Fraschetti nel sottolineare il ruolo importantissimo che, in questa koinè, ha avuto l'elemento greco ${ }^{23}$, passato attraverso le fasi dell'annichilimento e dell'omologazione.

In una prospettiva storico-religiosa questo rito arcaico e mai più ripetuto in seguito può spiegarsi solamente con l'annientamento rituale dei più potenti nemici di Roma in Italia.

La presa di Roma da parte dei Galli non portò solamente a questa innovazione in materia religiosa, ma anche a un culto che riguardava più strettamente la gens che ebbe il ruolo maggiore nella vicenda gallica. L'unica fonte al riguardo è ancora una volta Livio [5,46,2-3]:

sacrificium erat statum in Quirinali colle genti Fabiae. ad id faciendum C. Fabius Dorsuo Gabino cinctus sacra manibus gerens cum de Capitolio descendisset, per medias hostium stationes egressus nihil ad uocem cuiusquam terroremue motus in Quirinalem collem peruenit; ibique omnibus sollemniter peractis, eadem reuertens similter constanti uoltu graduque, satis sperans propitios esse deos quorum cultum ne mortis quidem metu prohibitus deseruisset, in Capitolium ad suos rediit, seu attonitis Galli miraculo audaciae seu religione etiam motis cuiusque haudquamquam neglegens gens est ${ }^{24}$.

22 Mazzarino 1944 (1922), in particolare 14-15 e 40-41, ma cfr. passim. "Già da più di un trentennio [siamo nel 1944] [... noi parliamo dello 'stato degli antichi Italici', presupponendo una koıv́́ culturale 'italica' in cui Etruschi, Latini, Greci d'Italia e Umbro-Oschi e via dicendo appaiono tutti a un tempo cointeressati e legati da vincoli di collaborazione culturale. S'intende che una tale koivì ha sfumature e gradi d'intensità diversissimi, e che accanto alla tendenza che potrebbe dirsi unificatrice c'è una tendenza centrifuga, che allontana e distrugge possibilità e fermenti di collaborazione culturale in Italia. Ma noi non possiamo più parlare di una Roma che si opponga all'Italia, costituendo come la tesi di un'antitesi in un sillogismo hegeliano» [14-15].

23 Fraschetti in Mazzarino 19922, x-xi.

24 «Era tradizione della gente Fabia celebrare periodicamente un sacrificio sul colle Quirinale. Per compierlo, Caio Fabio Dorsuone, con la toga cinta alla maniera dei 
Tuttavia la connessione tra Fabii e Galli non cessò con l'episodio di Brenno. Due anni dopo la vittoria sui Galli a Sentino (295 a.C.) venne infatti dedicato da quella gens a Roma sul Quirinale un tempio a Iuppiter Victor. È del tutto possibile l'ipotesi di Curti [2000, 85] che in quella seconda occasione i Fabii si fossero "ricordati" di quell'antico evento, inventandosi così una tradizione il cui scopo sarebbe stato quello di giustificare la presenza Fabia sul più sacro dei colli romani.

È tuttavia interessante rilevare come questi cambiamenti della religiosità tradizionale intervenuti all'indomani del sacco di Roma da parte dei Galli di Brenno abbiano avuto difficoltà a protrarre la loro efficacia oltre la fine del terzo secolo a.C. ${ }^{25}$. A partire da allora, infatti, i rapporti di forza in Italia erano definitivamente mutati a vantaggio di Roma e il metus Gallicus non poteva più generare mutamenti nel campo religioso, ma poteva ancora svolgere un ruolo rilevante nella destrutturazione del sistema istituzionale repubblicano.

Già gli antichi chiamarono Guerre cimbriche (bella Cimbrica) tutto il periodo compreso tra il 113 e il 101 [Piganiol, 379]. Si tratta di una definizione ampiamente enfatica, testimonianza già di per sé significativa della continua ricorrenza del metus. Non è il caso qui di affrontare il problema legato all'origine etnica delle popolazioni che scatenarono il metus cui pose rimedio Mario. Fatto sta che l'apparizione improvvi-

Gabini, portando in mano gli oggetti sacri, scese dal Campidoglio, e, dopo essere passato in mezzo ai posti di guardia nemici, senza minimamente lasciarsi impressionare dalle grida e dalle minacce di alcuno, giunse sul colle Quirinale: e celebrate qui solennemente tutte le sacre cerimonie, rifacendo la stessa strada con volto e passo ugualmente sicuri, fiducioso com'era di avere propizi gli dèi, il cui culto non aveva trascurato nemmeno per timore della morte, ritornò sul Campidoglio dai suoi, mentre i Galli erano, o sbalorditi dalla sorpresa per quell'atto di audacia, o anche sotto l'influenza del sentimento religioso, di fronte al quale nessun popolo rimane indifferente» (trad. M. Scandola).

25 Si noterà inoltre come questi cambiamenti non abbiano mai avuto una sanzione definitiva. Essi hanno dato luogo ad eventi religiosi ritenuti strumentalmente estranei alla mentalità religiosa romana e da questa respinta e limitata in una sfera di sostanziale estraneità. 
sa dei Cimbri nella storia avvenne già nel 117, con una dura sconfitta inferta al console Papirio Carbone a Noreia, località posta poco a sud di Vienna, nel Norico [Strabo 5,17]. Fu tuttavia il successivo disastro subito ad Arausium (Orange), lungo la valle del Rodano dal proconsole Gaio Servilio Cepione (105) ciò che indusse il senato a richiamare in tutta fretta dall'Africa il miglior generale a disposizione, il rude Gaio Mario, e a offrirgli tutta una serie di possibilità che a nessuno mai erano state concesse prima. Innanzi tutto Gaio Mario venne nominato ininterrottamente console dal $105 \mathrm{al}$ 100. Non è chiaro quando Mario abbia varato quelle riforme dell'esercito che avrebbero avuto conseguenze pesantissime nel futuro immediato. È possibile infatti che l'innovazione rappresentata dal sistematico arruolamento dei proletari nell'esercito risalga in effetti al consolato del 107 e non a quelli che seguirono quasi immediatamente in connessione con le guerre cimbriche. Fatto sta che la combinazione rappresentata dalla creazione di un esercito di proletari legato nelle sue fortune alla figura del comandante, unitamente alla continua iterazione per un lungo periodo del sommo imperio, produsse l'effetto di creare quei legami di fedeltà che univano il soldato al generale, piuttosto che allo Stato.

Il pericolo cimbrico (e teutonico) si rivelò in effetti ben poca cosa: dopo la lunga attesa dell'esercito mariano nella provincia di Gallia Narbonese, i Cimbri finalmente apparvero ancora una volta. Si diressero verso l'Italia da due direttrici diverse, ma vennero sconfitti dapprima nei pressi di Aquae Sextiae (Aix-en-Provence), poi, in maniera definitiva, in un vero e proprio massacro nei pressi di Vercelli (101). Tuttavia l'isteria scoppiata a Roma al loro primo apparire aveva ormai dato i suoi frutti: dopo il disastro di Orange «a Roma fu il panico. Il console Rutilio Rufo fece giurare ai giovani di non abbandonare l'Italia e i commissari vennero inviati nei porti per impedire l'imbarco degli uomini più giovani di 35 anni» [Piganiol 1967 378]. Le innovazioni che il Senato concesse a Mario per il terrore suscitato dai Cimbri - come s'è detto, l'opportunità di arruolare proletari e l'iterazione continuata del sommo potere 
- cambiarono per sempre la faccia e la portata dello scontro politico a Roma. Spinta dal terrore per i Cimbri Roma entrò nella fase in cui lo scontro politico divenne appannaggio di Warlords che si affronteranno al comando di eserciti sterminati.

\section{Bibliografia}

Bellen H. 1985, Metus Gallicus, metus Punicus: zum Furchtmotiv in der römischen Republik, Mainz: Akademie der Wissenschaften und der Literatur.

Briquel D. 2008, La prise de Rome par les Gaulois: lecture mythique d'un événement historique, Paris: PUPS.

Bruun C. 2000, "What every man in the street used to know»: M. Furius Camillus, Italic legends, and Roman Historiography, in Bruun C. (ed.), The Roman middle republic: politics, religion, and historiography : c. 400-133 B.C. : papers from a conference at the Institutum Romanum Finlandiae, September 11-12, 1998, Rome: Institutum Romanum Finlandiae, 41-68.

Cornell T., Rogers D. 1995, The beginnings of Rome: Italy and Rome from the Bronze Age to the Punic Wars (c. 1000-264 BC), London; New York: Routledge.

Curti E. 2000, From Concordia to the Quirinal: notes on religion and politics in mid-republican/hellenistic Rome, in Bispham E., Smith C. J. (eds.), Religion in archaic and republican Rome and Italy: evidence and experience, Chicago: Fitzroy Dearborn, 77-91.

Delfino A. 2009, L'incendio gallico : tra mito storiografico e realtà storica, "Mediterraneo Antico : economie, società, culture», XII (1/2): 339-360.

Dumézil G. 1973, Mythe et épopée. 3, Paris: Gallimard.

- 1974, La religion romaine archaïque, Paris: Payot.

Dumézil G., Strutynski U. 1980, Camillus: a study of Indo-European religion as Roman history, Berkeley: University of California Press.

Fraschetti A. 1981, Le sepolture rituali nel Foro Boario, in Torelli M. (ed.), Le delit religieux dans la cité antique: table ronde, Rome, 6-7 avril 1978, Roma: École française de Rome, 51-115.

Giardina A. 1997, L'Italia romana: storie di un'identità incompiuta, Roma-Bari: Laterza.

Mazzarino S. 1944 (1922), Dalla monarchia allo Stato repubblicano: ricerche di storia romana arcaica, Catania: G. Agnini.

- 1966, Il pensiero storico classico, Bari: Laterza.

- 1973, L'Impero romano, Roma-Bari: Laterza. 
- 1974, Antico, tardoantico ed èra costantiniana, Bari: Dedalo.

Mommsen T. 1879a, Die Gallische Katastrophe, in Römische Forschungen, Vol. II, Berlin: Weidmann, 297-381.

- 1879b, Fabius und Diodor, in Römische Forschungen, Vol. II, Berlin: Weidmann, 221296.

- 1899, Römisches strafrecht, Leipzig: Duncker \& Humblot.

Piganiol A. 1971, Le conquiste dei Romani, Milano: Il Saggiatore.

Richardson J. H. 2012, The Fabii and the Gauls Studies in historical thought and historiography in Republican Rome, Stuttgart: Franz Steiner Verlag.

Roberto U. 2012, Roma capta: il sacco della città dai Galli ai Lanzichenecchi, Roma-Bari: Laterza.

Russo F. 2012, L'Italia nella prospettiva romana (III secolo a.C.), «Studi classici e orientali», LVIII: 11-186.

Sordi M. 1960, I rapporti romano-ceriti e l'origine della civitas sine suffragio, Roma: L'Erma di Bretschneider.

Thornton J. 1998, Mísos Rhômaiôn o phóbos Mithridátou? Echi storiografici di un dibattito diplomatico, "Mediterraneo antico», I: 271-309.

Ungern-Sternberg J. von 2000, Eine Katastrophe wird verarbeitet: Die Gallier in Rom, in Bruun C. (ed.), The Roman middle republic: politics, religion, and historiography: $c$. 400-133 B.C. : papers from a conference at the Institutum Romanum Finlandiae, September 11-12, 1998, Rome: Institutum Romanum Finlandiae, 207-222.

Williams J. H. 2001, Beyond the Rubicon: Romans and Gauls in Republican Italy, Oxford: Oxford University Press.

Zecchini G. 2006, Migrazioni e invasioni in Polibio: il caso dei Celti, in Bertinelli Angeli M. G., Donati A. (eds.), Le vie della storia: migrazioni di popoli, viaggi di individui, circolazione di idee nel Mediterraneo antico : atti del II Incontro internazionale di storia antica (Genova 6-8 ottobre 2004), Roma: G. Bretschneider: 165-173.

- 2009, Le guerre galliche di Roma, Roma: Carocci. 